\title{
Peptide lipidation in lysophospholipid micelles and lysophospholipid-enriched membranes $\dagger$
}

\author{
Vian S. Ismail, $\ddagger^{a}$ Hannah M. Britt, (ID $\S^{a}$ Jackie A. Mosely (D) \\ and John M. Sanderson (D)*a
}

Received 17th April 2021, Accepted 16th July 2021

DOI: $10.1039 / d 1 f d 00030 f$

Acyl transfer from lipids to membrane-associated peptides is a well-documented process, leading to the generation of a lipidated peptide and a lysolipid. In this article, we demonstrate that acyl transfer from lysophosphatidylcholines (lysoPCs) to the peptide melittin also occurs, both in micelles of pure lysolipid and in lipid/lysolipid mixtures. In the case of bilayers containing lysolipids, acyl transfer from the lysolipid is marginally favoured over transfer from the lipid. In pure bilayers of saturated lipids, the introduction of even small amounts of lysolipid appears to significantly increase the reactivity towards lipidation.

\section{Introduction}

It is now well established that acyl transfer can potentially occur from esterbased lipids to any molecules that are able to interact with lipid membranes and possess suitable acceptor groups. ${ }^{1}$ Examples of molecules known to undergo this process, termed intrinsic lipidation, include low molecular weight organics ${ }^{2,3}$ and peptides. ${ }^{4-7}$ Suitable acceptor groups include amino groups and alcohols. When the donor is a lipid, a lysolipid byproduct is formed alongside the lipidated acceptor, leading to the formation of mixed lipid/lysolipid systems (Fig. 1a). Many of these acyl transfer experiments have been conducted in vitro in liposomes composed of synthetic lipids, for which the origin of the acyl group is unambiguous. Early studies on intrinsic lipidation were conducted using melittin, obtained synthetically to avoid complications from the presence of

\footnotetext{
${ }^{a}$ Chemistry Department, Durham University, Durham, DH1 3LE, UK. E-mail: j.m.sanderson@durham.ac.uk ${ }^{b}$ National Horizons Centre, School of Health \& Life Sciences, Teesside University, Darlington, DL1 1HG, UK. E-mail:j.mosely@tees.ac.uk

$\dagger$ Electronic supplementary information (ESI) available. See DOI: 10.1039/d1fd00030f

\$ Current address: Chemistry Department, Soran University, Kurdistan Region, Iraq, vian.esmaeil@ soran.edu.iq.

§ Current address: Institute of Structural \& Molecular Biology, University College London, Gower Street, London, WC1E 6BT, UK, h.britt@ucl.ac.uk.
} 
a

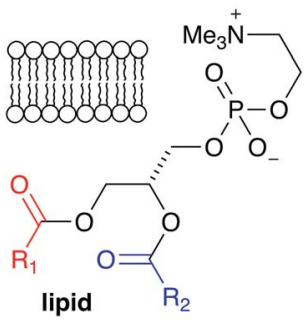

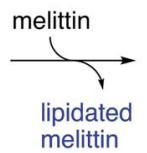

\section{b}

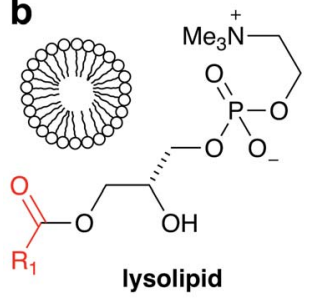
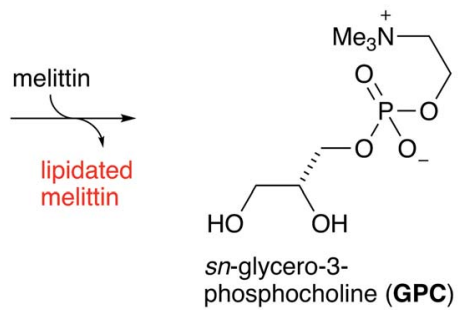

C H-GIGAVLLKVLTTGLPALISWIIKRKRQQ-NH ${ }_{2}$

Fig. 1 Summary of the reactivity of melittin with lipids: (a) reaction with ester-linked diacyl lipids leads to the generation of lipidated melittin and a lysolipid byproduct; (b) reaction with lysolipids leads to the formation of lipidated melittin and the corresponding deacylated glycerol derivative GPC; (c) sequence of melittin, with the main lipidation residues highlighted in bold and the minor sites underlined.

phospholipase $\mathrm{PLA}_{2}$ found in melittin extracted from bee venom. It was found that melittin undergoes ready intrinsic lipidation in membranes composed of unsaturated phosphatidylcholine (PC) based lipids. The reaction was generally favoured at the N-terminal amino group of the peptide, but significant lipidation of Lys23 was also noted, alongside minor amounts of lipidation of the side chains of Lys21, Lys7 and Ser18 (Fig. 1). ${ }^{6,7}$ Lipidation activity was generally influenced by the presence of other lipid components such as PE, PS or PG, but the manner of the influence was very composition dependent. ${ }^{5,6}$ More recent work has further demonstrated the complexity of this process, as cholesterol incorporation into PC membranes both significantly increases lipidation activity, despite decreasing the affinity of the peptide for the membrane, and changes the relative reactivity at different sites on the peptide. Moreover, membranes composed of saturated PC lipids are found to be unreactive in the absence of cholesterol. ${ }^{4}$

In a number of these experiments, there appears to be generally little selectivity between transfer from the $s n-1$ and $s n-2$ acyl groups of the lipids, especially at longer timescales after peptide/lipid mixing. In the initial periods after mixing, however, $s n-1 / s n-2$ selectivity is sometimes apparent in liquid chromatography-mass spectrometry (LC-MS) analyses, which may reflect minor differences in product ionisability near the detection limit, or alternatively may reflect a significant difference in reactivity between lipids and lysolipids. Small 
amounts of lysolipids may be pre-existing as a consequence of hydrolysis, or formed in the membrane as the by-products of lipidation. In the latter case, as lysolipids are potential acyl group donors, any intrinsic preference for reaction at the $s n-1$ and $s n-2$ positions of the lipid may be masked if the lysolipid is more reactive than the lipid.

It is therefore of fundamental importance to determine the level of reactivity of lysolipids towards membrane-associated peptides. Given the sensitivity of intrinsic lipidation to membrane composition, the generation of lysolipids in situ is also expected to have a significant impact on the extent and selectivity of lipidation. This article describes measurements of melittin lipidation undertaken over a range of surfactant compositions, ranging from pure lysolipid, through lysolipid/lipid mixtures, to pure lipids.

\section{Methods}

\section{Materials}

Synthetic melittin ( $\geq 97 \%$ by HPLC) and bee venom melittin ( $>85 \%$ by HPLC) were obtained from Sigma-Aldrich, Dorset, UK. A stock solution of the peptide at an approximate concentration of $1 \mathrm{mg} \mathrm{ml}^{-1}$ was prepared in water and used fresh. The concentration of peptide was more accurately determined before use by absorbance measurements $\left(\varepsilon\right.$ at $\left.250 \mathrm{~nm}=5500 \mathrm{M}^{-1} \mathrm{~cm}^{-1}\right) .^{7,8}$ Authentic samples of $N^{1}$ - and $\mathrm{K}^{23}$-oleoyl and palmitoyl melittin were obtained from Almac Group (Craigavon, UK) at $>95 \%$ purity by HPLC.

1,2-Dioleoyl-sn-glycero-3-phosphocholine (DOPC) and 1,2-dipalmitoyl-snglycero-3-phosphocholine (DPPC) were obtained from Sigma-Aldrich, Dorset, UK. 1-Oleoyl-2-hydroxy-sn-glycero-3-phosphocholine (OPC) and 1-palmitoyl-2-hydroxy$s n$-glycero-3-phosphocholine (PPC) were obtained from Avanti Polar Lipids, USA. Water was purified using a Milli-Q Direct Q system from Millipore (Millipore (UK) Ltd) to give a resistivity of $\geq 18 \mathrm{M} \Omega \mathrm{cm}^{-2}$. Stock dispersions of lysoPCs in water were prepared at a concentration of $1.3 \mathrm{mM}$ in aqueous buffer $(90 \mathrm{mM} \mathrm{NaCl}$, $10 \mathrm{mM}$ sodium phosphate, $\mathrm{pH}$ 7.4). Other solvents and reagents were obtained from Fisher Scientific, UK.

\section{Liposome preparation}

Liposomes were prepared by concentrating a solution of lipid $(1 \mathrm{mg})$ in chloroform $(100 \mu \mathrm{l})$ in vacuo to form a thin film. The lipid film was hydrated with $1 \mathrm{ml}$ of aqueous medium containing $90 \mathrm{mM} \mathrm{NaCl}$ buffered with $10 \mathrm{mM}$ sodium phosphate at $\mathrm{pH} 7.4$ and mixed thoroughly before being subjected to five freeze thaw cycles and extruded 10 times through a $100 \mathrm{~nm}$ laser-etched polycarbonate membrane (Whatman) at $50{ }^{\circ} \mathrm{C}$ under a stream of nitrogen using a thermobarrel extruder (Northern Lipids, Burnaby, Canada).

\section{Lipidation experiments}

Standard lipidation conditions employed a buffer of $90 \mathrm{mM} \mathrm{NaCl}$ buffered with $10 \mathrm{mM}$ sodium phosphate at $\mathrm{pH} 7.4$ at $37^{\circ} \mathrm{C}$. Mixtures of lysolipid and lipid were prepared by adding a dispersion of the lysolipid in buffer to an extruded dispersion of unilamellar liposomes, followed by agitation. Melittin was then added to the mixture to give a final peptide concentration of $50 \mu \mathrm{m}$ and a lipid 
concentration of $0.65 \mathrm{mM}$, and the sample was agitated. Samples for analysis were diluted by a factor of 5 in water. The times given in the text refer to the time postmixing after melittin addition.

\section{Liquid chromatography-mass spectrometry (LC-MS)}

Separations were performed using an Xbridge C18 column $(3.5 \mu \mathrm{m}$ particle size, $2.1 \mathrm{~mm}$ internal diameter, $100 \mathrm{~mm}$ length; Waters UK, Manchester, UK). Typical analyses used a $5 \mu \mathrm{l}$ sample injection at a flow rate of $200 \mu \mathrm{lmin}^{-1}$ with a gradient of mobile phases A $(0.1 \%(\mathrm{v} / \mathrm{v})$ formic acid (FA) in water), B $(0.1 \%(\mathrm{v} / \mathrm{v})$ FA in acetonitrile) and $\mathrm{C}(0.1 \%(\mathrm{v} / \mathrm{v}) \mathrm{FA}$ in methanol) as follows: $95 \% \mathrm{~A}: 5 \% \mathrm{~B}$ to $5 \%$ A : $95 \%$ B over $10 \mathrm{~min}$, followed by $5 \mathrm{~min}$ at $100 \% \mathrm{~B}$ and $15 \mathrm{~min}$ at $100 \% \mathrm{C}$, plus 5 min re-equilibration at $95 \% \mathrm{~A}: 5 \% \mathrm{~B}$.

Positive ion mass spectra were recorded over $m / z$ 200-2000 using a LTQFT mass spectrometer equipped with a $7 \mathrm{~T}$ magnet (ThermoFisher Corp., Bremen, Germany). Electrospray ionisation (ESI) was used to generate positive ions using the following conditions: source voltage $4.0 \mathrm{kV}$, capillary voltage $30.0 \mathrm{~V}$, capillary temperature $350{ }^{\circ} \mathrm{C}$ and tube lens $100.0 \mathrm{~V}$. The auxiliary gas flow and sweep gas flow were set at 5.0 arbitrary units and the nitrogen sheath gas flow at 15.0 arbitrary units. Collision-induced dissociation experiments were performed entirely within the linear ion trap with a fixed isolation window of $4 \mathrm{~m} / \mathrm{z}$, using helium as a collision gas and an optimized normalized collision energy level of $25 \%$. Spectra were analysed using XcaliburQualBrowser version 2.0.7 (Thermo Fisher Scientific Inc.) and processed using the embedded program Qual Browser.

\section{Peptide CMC measurements}

A peptide stock solution of approximately $1 \mathrm{mg} \mathrm{ml} \mathrm{m}^{-1}$ in water was added sequentially to a solution of Rhodamine $6 \mathrm{G}(0.08 \mu \mathrm{M}, 45 \mu \mathrm{l})$ in water in a $40 \mu \mathrm{l}$ ultra-micro quartz cuvette to give peptide concentrations in the range $1 \times 10^{-6} \mathrm{~m}$ to $1 \times 10^{-4} \mathrm{M}$. Following each addition, fluorescence emission was measured in the range $500 \mathrm{~nm}$ to $600 \mathrm{~nm}$, with an excitation wavelength of $480 \mathrm{~nm} .{ }^{9,10}$ Emission intensities at $550 \mathrm{~nm}$ were corrected for dilution.

\section{Melittin reactivity with lysolipids}

Initial experiments were conducted by mixing synthetic melittin with lysophosphatidylcholine (lysoPC) micelles. Experiments were conducted using a lysoPC with either a saturated acyl chain (palmitoyl-sn-glycero-3-phosphocholine, PPC), an unsaturated acyl chain (oleoyl-sn-glycero-3-phosphocholine, OPC) or a $1: 1$ mixture of the two. These glycerol-based lysolipids all exist as a mixture of 1- and 2 -acyl isomers in equilibrium, with the 1-acyl being predominant. In all cases (Fig. 2), lipidated melittin products were readily detectable after $24 \mathrm{~h}$ and were the predominant melittin species present after $168 \mathrm{~h}$.

Two features of the lipidation in the lysoPC micelles were particularly striking. First, there was a shift in selectivity towards lipidation on the Lys23 side chain in OPC micelles (Fig. 2b: ii > i), rather than the N-terminal amino group preference found for PPC (Fig. 2d: vii > viii) and mixed OPC/PPC micelles (Fig. 2f: I > ii and vii $>$ iii), and observed previously with lipids. ${ }^{4-6}$ Second, the levels of melittin bearing 

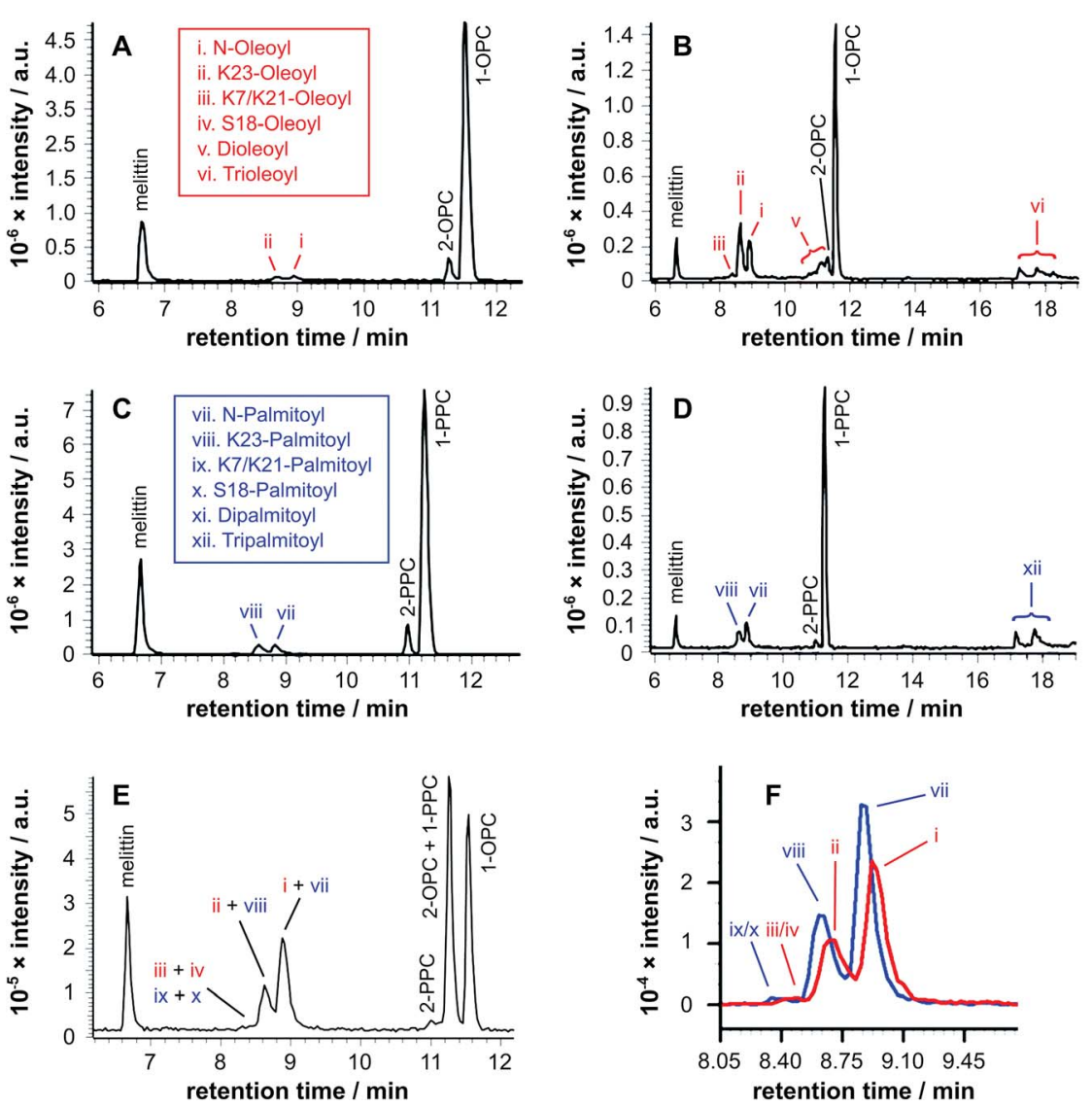

Fig. 2 LC-MS analysis (ESI-FTICR-MS (LTQFT), $C_{18}$ ) of synthetic melittin incubated with lysolipid micelles at $37{ }^{\circ} \mathrm{C}$ in buffer $(10 \mathrm{mM} \mathrm{NaHCO} / 90 \mathrm{mM} \mathrm{NaCl})$ at $\mathrm{pH} 7.4$. (a) $1 / 2-$ Oleoyl-sn-glycero-3-phosphocholine (OPC), 24 h; (b) OPC, 168 h; (c) 1/2-palmitoyl-snglycero-3-phosphocholine (PPC), 24 h; (d) PPC, 168 h; (e) $1: 1 \mathrm{PPC} / \mathrm{OPC}, 168 \mathrm{~h}$; (f) extracted ion chromatograms (EICs) from spectrum (e) for $\mathrm{m} / \mathrm{z} 772.00$ and 778.51 , corresponding to palmitoyl and oleoyl melittin respectively $(z=4)$. MS data for the lipidated products are in Fig. S1 to S4 and Tables S1 to S4. $\dagger$

multiple acyl groups was significant, particularly in OPC micelles, in which melittin lipidated with both two and three acyl groups was detected. Tripleacylated melittin was also observed in PPC micelles, although curiously the double palmitoylated species was absent, which could be accounted for by the faster addition of the third acyl group in PPC micelles compared to the addition of the second.

\section{Melittin reactivity in lysolipid/lipid mixtures}

In order to examine qualitatively the relative reactivity of PC-based lysolipids and lipids with melittin, a series of experiments was conducted using a range of systems composed of lipids, lysolipids, and mixtures of the two. The experiments were undertaken using lipids with the same acyl group at the $s n-1$ and $s n$ - 
2 positions and a lysoPC with a different acyl chain in order to permit transfer from the lysolipid and the lipid to be distinguished. For the 1:1 mixtures of lysolipid and lipid, melittin analogues modified with single, double, and triple acyl modifications were observed after extended time periods (Fig. 3). An interesting feature of the product profiles for the double acylated products is the observation of similar product ratios regardless of whether they are formed in PPC/DOPC or OPC/DPPC mixtures. For example, the product ions for melittin modified with oleoyl + palmitoyl and $2 \times$ oleoyl or $2 \times$ palmitoyl are of similar relative abundance in both mixtures (Fig. S2 $\dagger$ ). The same observations apply for the triple acylated melittin (Fig. S3 and S4 $\dagger$ ). These observations suggest that there is little selectivity between lysolipids and lipids when it comes to the addition of an extra acyl group to a melittin molecule that is already lipidated.

Tandem mass spectrometry analysis (Fig. S5 to S19 and Tables S5 to S32†) revealed that the predominant double-acylated product arose from lipidation at the both the $\mathrm{N}$-terminus and at $\mathrm{K} 23$, in line with the relative abundance of the single acyl modifications to these sites. The second most abundant double modification was to the side chains of K23 and K7. Modification of the K7 side chain was usually of a relatively low abundance, suggesting that the presence of
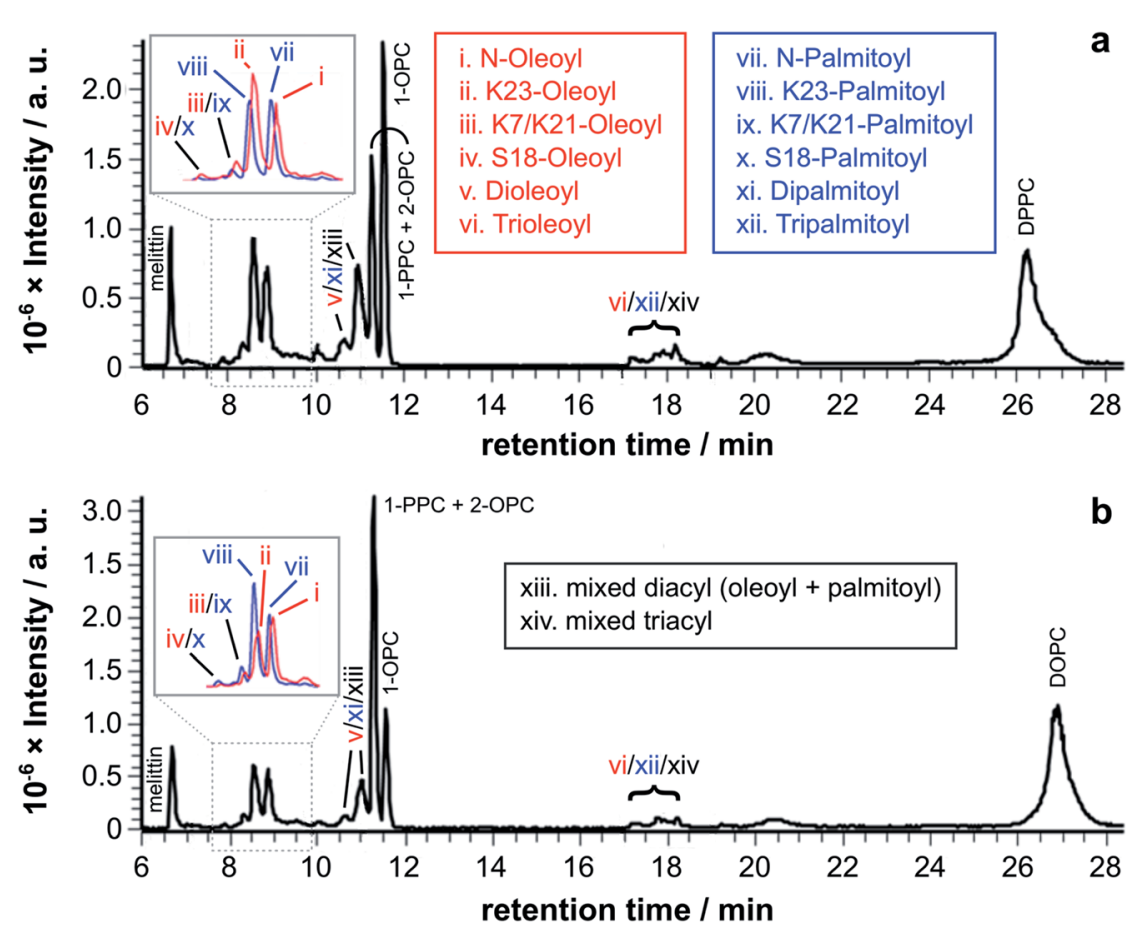

Fig. 3 LC-MS analysis of synthetic melittin incubated with 1: 1 (molar) mixtures of lysolipid and lipid at $37^{\circ} \mathrm{C}$ in buffer $(10 \mathrm{mM} \mathrm{NaHCO} / 90 \mathrm{mM} \mathrm{NaCl})$ at $\mathrm{pH}$ 7.4. (a) OPC/1,2dipalmitoyl-sn-glycero-3-phosphocholine (DPPC); (b) PPC/1,2-dioleoyl-sn-glycero-3phosphocholine (DOPC). The insets show EICs for $\mathrm{m} / \mathrm{z} 772.00$ and 778.51 , corresponding to palmitoyl and oleoyl melittin respectively $(z=4)$. MS data for the lipidated products are in Fig. S1 to S4 and Tables S1 to S4.† 
a single fatty acyl modification changes the reactivity at other sites, most likely as a consequence of a change in the interfacial positioning of the peptide. Two products were also tentatively identified as arising from lipidation at the $\mathrm{N}$ terminus and the side chain of either R22 or R24. These materials had the retention time characteristics of the double-acylated materials, but intact molecular ions were challenging to observe. Acyl modifications to arginine side chains would be expected to be highly labile and fragment easily in the source. Modification at other nearby sites, K21 and K23, could be ruled out as these products were identified elsewhere in the chromatogram.

A more extended series of lysolipid/lipid mixtures was examined. The series as a whole covered lipid bilayers, bilayers containing lysolipids, lysolipid micelles containing lipids, mixed micelle/lipid systems and micelles (Fig. 4).

For mixtures of OPC with DPPC (Fig. 4a-e), oleoyl transfer was always favoured to the side chain of Lys 23 of the peptide (peak ii), regardless of whether the mixture was a micelle or bilayer containing detergent. The relative abundance of palmitoylated products arising from transfer to the $\mathrm{N}$-terminal amino group (peak
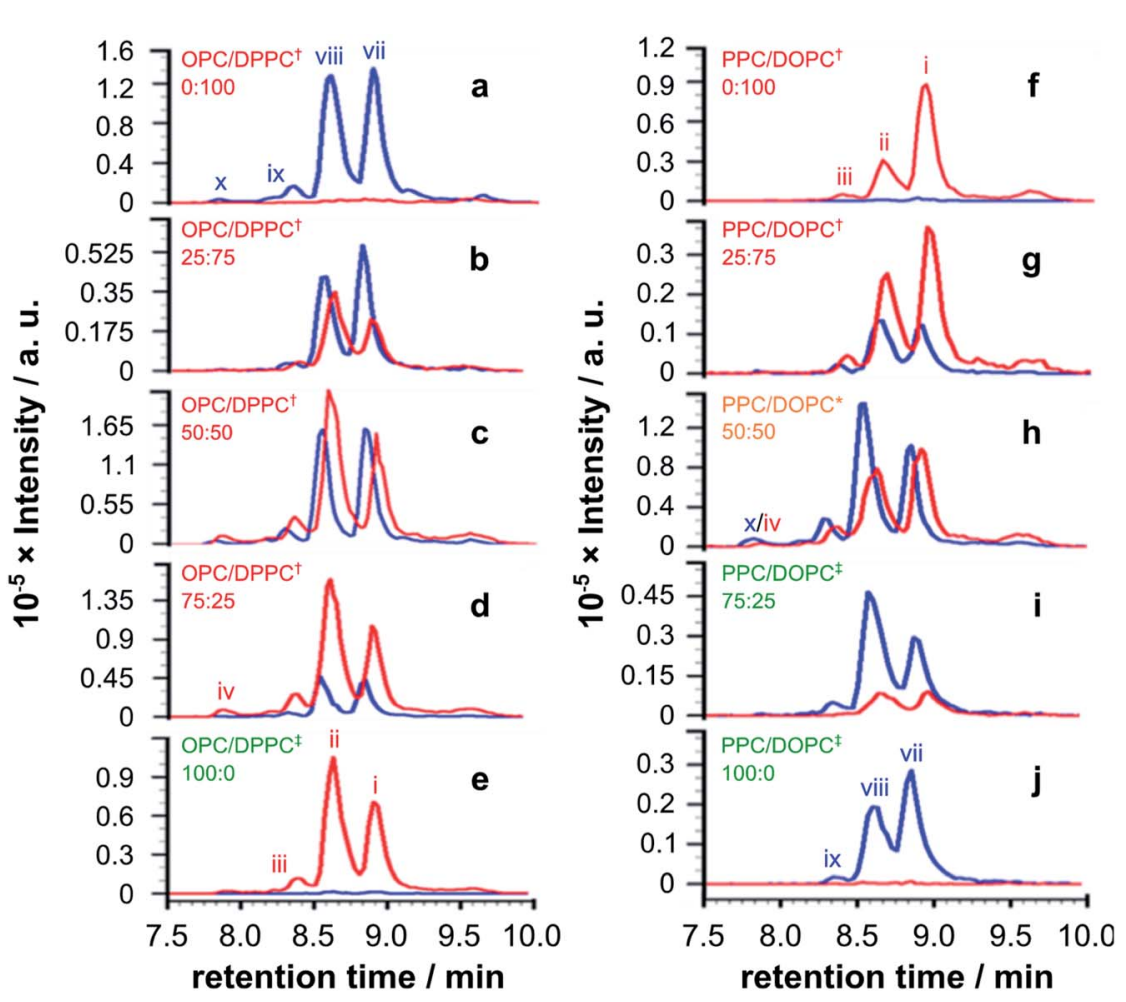

Fig. 4 EICs for $\mathrm{m} / \mathrm{z} 772.00$ and 778.51 , corresponding to palmitoyl and oleoyl melittin respectively $(z=4)$, from LC-MS analyses of synthetic melittin incubated for $168 \mathrm{~h}$ with mixtures of the lysolipid and lipid at $37{ }^{\circ} \mathrm{C}$ in buffer $(10 \mathrm{mM} \mathrm{NaHCO} 3 / 90 \mathrm{mM} \mathrm{NaCl})$ at $\mathrm{pH}$ 7.4. (a to e) DPPC + OPC; (f to j) DOPC + PPC. The initial molar ratio of lysolipid to lipid is indicated on each trace. Traces are labeled according to their expected phase behavior on the basis of literature precedents:11-14 bilayer \pm lysolipid $(\dagger)$; mixed bilayer/detergent $(*)$; or micelles $(\$)$. Peak annotations are identified in Fig. 2 and 3 . The corresponding data after $48 \mathrm{~h}$ are given in Fig. $\mathrm{S} 20 . \dagger$ 
vii) or the side chain of Lys23 (peak viii) was always around 50/50. In previous work, melittin was found to be unreactive in DPPC membranes. ${ }^{4}$ However, this was not the case in the work reported here (Fig. 4a). In seeking to account for this difference it was found that small levels of OPC were detectable in these DPPC samples. Blank LC-MS runs acquired immediately before the DPPC samples were analysed did not reveal any detectable OPC, so the likely source of this material in the DPPC experiments is presumably a small amount of carry over from previous work in the same apparatus. On the basis of this finding, we hypothesise that the presence of even small quantities $(<1 \mathrm{~mol} \%)$ of lysolipid in a bilayer with saturated acyl chains is sufficient to perturb structure and packing to the extent that lipidation becomes feasible. This hypothesis is in line with previous work that has demonstrated the significant instability of DPPC membranes containing $1 \mathrm{~mol} \%$ OPC. ${ }^{11}$ If this bilayer disruption can be proven to extend to lower concentrations of lysolipid, it will have significant ramifications for bilayer stability in scenarios where membrane damage can occur, as even small amounts of hydrolysis or oxidation may facilitate other kinds of reactivity. For mixtures of PPC with DOPC (Fig. $4 \mathrm{f}-\mathrm{j}$ ), oleoyl transfer from the lipid to the N-terminal amino group of the peptide was always preferred (peak i), in line with previous experiments. ${ }^{4-7}$ Palmitoyl transfer, however, exhibited more complex behavior. In systems containing micelles and lipids (Fig. 4h, i), transfer from the lysolipid to the side chain of Lys23 was always preferred (peak viii). In pure lysolipid micelles however (Fig. 4j), reaction at the N-terminal amino group of melittin was favoured (peak vii). In bilayers containing lysolipid (Fig. 4g), products formed by transfer from the lysolipid to Lys23 and the $\mathrm{N}$-terminal amino group were of similar relative abundance.

The data in Fig. 4 show only the regions of the analyses corresponding to melittin modified by a single acyl group at a single time point. Given the low selectivity observed for the subsequent transfer of acyl groups to a melittin molecule that has already been lipidated (Fig. 3), the product distributions in Fig. 4 are likely to reflect the selectivity for the addition of the first acyl group, with the caveat that any initial deviations in the overall reaction rate immediately following peptide addition to the membranes will not be captured. In each sample in Fig. 4, the relative proportion of melittin lipidated by the transfer of a single acyl group from the lysolipid or the lipid is broadly in line with the molar ratio of these lipids, consistent with each (diacyl) lipid reacting only once. However, comparison of the data for both $1: 1$ samples (Fig. $4 \mathrm{c}$ and $\mathrm{h}$ ) suggests that lysolipid reactivity with melittin is marginally higher than lipid reactivity. This is also supported by the comparison of each $25: 75$ mixture with its corresponding $75: 25$ mixture, such as Fig. $4 \mathrm{~g}$ and $4 \mathrm{i}$, in which the proportion of product formed by acyl transfer from the lysolipid is higher in the $25: 75$ mixture than transfer from the lipid in the $72: 25$ mixture.

Overall, the pattern of product formation is indicative of a marginally higher reactivity of melittin towards lysolipids than lipids. Product distributions, in terms of the relative levels of acyl transfer to different residues in the melittin sequence, are much more sensitive to bilayer composition. This sensitivity to bilayer composition most likely reflects corresponding differences in the interfacial orientation and location of melittin in each lipid mixture. 


\section{Reactivity of bee venom melittin in PC/PS mixtures}

Melittin extracted from bee venom (BVM) is usually obtained in the presence of phospholipase A2 $\left(\mathrm{PLA}_{2}\right)$ as a contaminant. For most applications, the level of $\mathrm{PLA}_{2}$ activity can be minimised by the use of a $\mathrm{Ca}^{2+}$ chelator such as ethylene glycol-bis( $\beta$-aminoethyl ether)- $N, N, N^{\prime}, N^{\prime}$-tetraacetic acid (EGTA). For the work reported here, however, it was desirable to examine whether $\mathrm{PLA}_{2}$ activity, leading to the formation of lysolipids, was able to influence the lipidation process. Therefore, BVM was added to the liposomes in the absence of any metal chelator. In the case of the pure PC membranes, the major product, by a considerable margin, resulted from the transfer of the $s n-1$ chain from the lysolipid form by $\mathrm{PLA}_{2}$ activity: oleoyl in the case of 1-oleoyl-2-palmitoyl-sn-glycero-3-phosphocholine (OPPC) and palmitoyl for 1-palmitoyl-2-oleoyl-sn-glycero-3-phosphocholine (POPC). For membranes composed of mixtures of PC with phosphatidylserine (PS) lipids (Fig. 5), the major lipidation product arose, as expected, by the transfer of the $s n-1$ acyl chain of the PC component of the mixture.

In a similar manner, the level of stearoyl transfer from SLPS was greater than that of linoleoyl transfer, particularly in the case of the OPPC/SLPS mixture, reflecting the reactivity of bee venom PLA 2 with SLPS. ${ }^{15}$

These data are consistent with significant reactivity towards the lysolipids formed by $\mathrm{PLA}_{2}$ activity, assuming that the rate of lipase-catalysed hydrolysis is faster than that of lipidation, which is a reasonable assumption based on literature precedents. ${ }^{\mathbf{1 6 , 1 7}}$

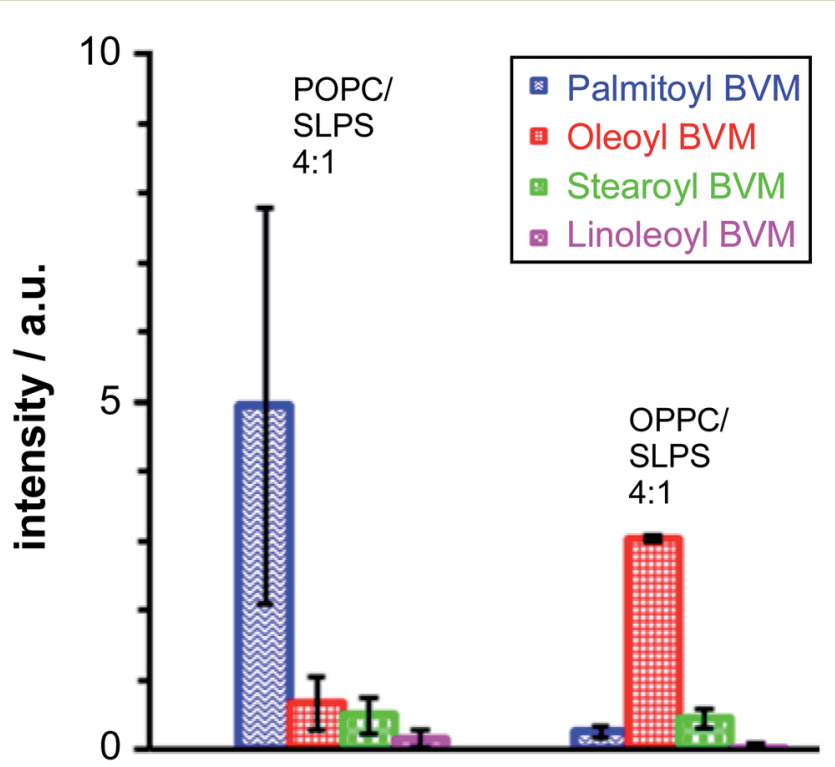

Fig. 5 Peak areas for singly lipidated melittin products following LC-MS analysis of mixtures of bee venom melittin (BVM) with POPC/1-stearoyl-2-linoleoyl-sn-glycero-3phosphoserine (SLPS; $4: 1$ ) and OPPC/SLPS (4:1) liposomes incubated for $24 \mathrm{~h}$ at $37^{\circ} \mathrm{C}$, $\mathrm{pH}$ 7.4. Errors are plotted as the SEM of the normalised peak area $(n=2)$. Normalisation of $\mathrm{BVM}$ single acylation was performed relative to the non-acylated BVM in the trace. 


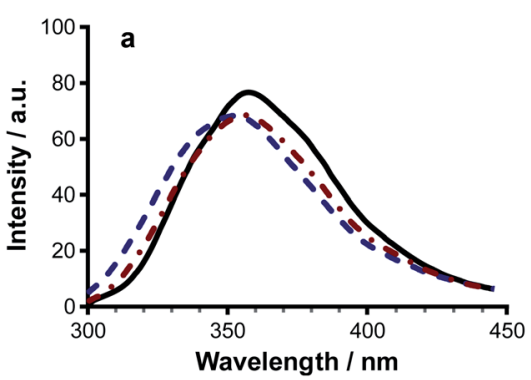

Fig. 6 The effects of lipidation on melittin structure. (a) Fluorescence emission ( $\lambda_{\text {ex }} 280$ $\mathrm{nm}$ ) for melittin (solid black line), $N^{1}$-palmitoyl melittin (dotted and dashed red line) and $N^{1}$ oleoyl melittin (dashed blue line). (b) CD spectra of $N^{1}$-palmitoyl melittin (red); $\mathrm{K}^{23}$-palmitoyl melittin (orange); $N^{1}$-oleoyl melittin (blue); and $\mathrm{K}^{23}$-oleoyl melittin (green). Authentic samples of peptides acylated at the $\mathrm{N}$-terminus and $\mathrm{K} 23$ side chain were obtained commercially.

\section{The effects of lipidation on peptide structure}

Melittin is known to be unfolded in solution as a monomer, forming a folded helix in the membrane and a tetramer at high concentrations. ${ }^{18-22}$ Lipidation has the potential to disrupt the balance between these structural forms, or to push the peptide towards additional conformational states. Authentic samples of lipidated melittin exhibited fluorescence and CD spectral profiles typical of a folded helical form, with blue shifted Trp emission (Fig. 6a) and negative ellipticity minima at approximately $210 \mathrm{~nm}$ and $225 \mathrm{~nm}$ (Fig. 6a).

Modifications at both the N-terminus and side chain of Lys23 were able to induce folding of the peptide in solution. Fluorescence measurements of solutions of acylated melittin prepared in the presence of Rhodamine $6 \mathrm{G}^{23}$ indicated that the acylated peptides are able to form micelles, with critical micelle concentrations of $\leq 10 \mu \mathrm{M}$ (Fig. S22 $\dagger$ ). Micelle formation may be a factor in driving the adoption of the peptide secondary structure.

\section{Conclusions}

As a whole, the data demonstrate that melittin is readily lipidated by acyl transfer from lysophosphatidylcholines (lysoPCs) in all of the systems studied: micelles, PC bilayers containing lysolipid, and mixed lipid/micelle dispersions. The reactivity of the peptide with lysoPCs in these mixtures is marginally higher than that with lipids and leads to the modification of melittin lipidated at a number of sites, predominantly the N-terminus and the side chain of Lys23. In mixtures of lysoPCs without lipid, there is some selectivity according to the nature of the acyl group of the lysolipid, with palmitoyl transfer being favoured over oleoyl. Although the same range of products is formed by reaction with either lipids or lysolipids, the ratio of lipidation at the $\mathrm{N}$-terminus and Lys23 is sensitive to the composition of the mixture, favouring the $\mathrm{N}$ terminus in systems rich in DOPC and Lys23 in most other mixtures apart from $100 \%$ PPC. These changes in the product profile are likely to be a consequence of the composition of the heterogeneous phase influencing both the 
preferred depth and orientation of melittin binding in the interface and the positioning of lysolipid and lipid acyl groups.

Melittin is known to associate favourably with regions of high positive curvature, ${ }^{24}$ a tendency that may be associated with the ability of the peptide to form pores. As membrane remodelling and pore formation are both expected to occur faster than lipidation, it is therefore possible that the changes in the product profile result from lipid segregation and aggregation. Such phenomena were not probed here, however.

In general terms, how the interplay between peptide structure and membrane composition influences lipidation, both in selectivity and overall reactivity, are matters for further discussion.

For membranes composed of saturated lipids such as DPPC, for which the lipidation activity of melittin is normally low, the presence of even a small amount of lysolipid appears to be sufficient to promote reactivity. The mechanism by which this occurs is unclear, but it is likely to result from a disruption to lipid packing that would, for example, enable better water penetration into the interface. The presence of interfacial water has the potential to facilitate the formation of products from intermediates in the acylation reaction. ${ }^{6,25,26}$ Should this be the case, other scenarios that can facilitate water penetration into the membrane, such oxidative damage, should also produce increased rates of acyl transfer reactions from lipids to suitable acceptors. The role that physical and chemical stresses play in promoting intrinsic lipidation is worthy of further consideration.

For melittin, the addition of a fatty acyl group at either of the two main sites of modification by transfer from lipids is sufficient to promote the adoption of a secondary structure in the peptide and drive micellisation. At low concentrations of acylated peptide, it is likely that acylation both increases the affinity of the peptide for the heterogeneous phase and modifies peptide orientation in the interface. These changes in affinity and orientation may account for the extensive formation of peptides modified with multiple acyl groups in lysolipid micelles. At higher peptide concentrations in all systems, the formation of peptide-containing micelles is likely. It is notable that the formation of micelles following the addition of melittin to lipid membranes has been documented. ${ }^{27,28} \mathrm{~A}$ key issue for debate is whether lipidation is able to steer the folding of peptides into particular conformations with adverse biological activity. In this regard, it has been proposed that by driving secondary structure formation and tethering peptides to the membrane, lipidation offers a route for facilitating the nucleation of amyloid fibrils. ${ }^{1}$

\section{Author contributions}

JMS and JAM conceived of and supervised the project. VSE performed the lipidation work with melittin. VSE and JMS analysed the data. HMB performed the CMC work with authentic samples of acylated melittin and analysed the data. The manuscript was written by JMS, with contributions from all authors.

\section{Conflicts of interest}

There are no conflicts of interest to declare. 


\section{Acknowledgements}

The authors thank the Ministry of Higher Education and Scientific Research (grant number 1391) of the Kurdistan Regional Government for support to VSE, and the Engineering and Physical Sciences Research Council (EP/M506321/1) for support to HMB.

\section{References}

1 J. M. Sanderson, BioEssays, 2020, 42, e1900147.

2 H. M. Britt, A. S. Prakash, S. Appleby, J. A. Mosely and J. M. Sanderson, Sci. Adv., 2020, 6, eaaz8598.

3 H. Britt, C. A. García-Herrero, P. W. Denny, J. A. Mosely and J. M. Sanderson, Chem. Sci., 2019, 10, 674-680.

4 H. M. Britt, J. A. Mosely and J. M. Sanderson, Phys. Chem. Chem. Phys., 2019, 21, 631-640.

5 R. H. Dods, B. Bechinger, J. A. Mosely and J. M. Sanderson, J. Mol. Biol., 2013, 425, 4379-4387.

6 R. H. Dods, J. A. Mosely and J. M. Sanderson, Org. Biomol. Chem., 2012, 10, 5371-5378.

7 C. J. Pridmore, J. A. Mosely, A. Rodger and J. M. Sanderson, Chem. Commun., 2011, 47, 1422-1424.

8 C. N. Pace, F. Vajdos, L. Fee, G. Grimsley and T. Gray, Protein Sci., 1995, 4, 2411-2423.

9 P. P. Bonsen, G. H. de Haas, W. A. Pieterson and L. L. van Deenen, Biochim. Biophys. Acta, Lipids Lipid Metab., 1972, 270, 364-382.

10 P. Becher, J. Phys. Chem., 1962, 66, 374-375.

11 C. J. Van Echteld, B. de Kruijff, J. G. Mandersloot and J. De Gier, Biochim. Biophys. Acta, Biomembr., 1981, 649, 211-220.

12 J. R. Henriksen, T. L. Andresen, L. N. Feldborg, L. Duelund and J. H. Ipsen, Biophys. J., 2010, 98, 2199-2205.

13 D. V. Zhelev, Biophys. J., 1998, 75, 321-330.

14 D. Needham and D. V. Zhelev, Ann. Biomed. Eng., 1995, 23, 287-298.

15 E. A. Dennis, J. Cao, Y. H. Hsu, V. Magrioti and G. Kokotos, Chem. Rev., 2011, 111, 6130-6185.

16 L. Feng, K. Manabe, J. C. Shope, S. Widmer, D. B. DeWald and G. D. Prestwich, Chem. Biol., 2002, 9, 795-803.

17 F. Ghomashchi, Y. Lin, M. S. Hixon, B. Z. Yu, R. Annand, M. K. Jain and M. H. Gelb, Biochemistry, 1998, 37, 6697-6710.

18 I. Constantinescu and M. Lafleur, Biochim. Biophys. Acta, Biomembr., 2004, 1667, 26-37.

19 C. E. Dempsey, Biochim. Biophys. Acta, Rev. Biomembr., 1990, 1031, 143-161.

20 W. Wilcox and D. Eisenberg, Protein Sci., 1992, 1, 641-653.

21 P. Yuan, P. J. Fisher, F. G. Prendergast and M. D. Kemple, Biophys. J., 1996, 70, 2223-2238.

22 J. F. Faucon, J. Dufourcq and C. Lussan, FEBS Lett., 1979, 102, 187-190.

23 H. S. Courtney, W. A. Simpson and E. H. Beachey, Infect. Immun., 1986, 51, 414-418. 
24 P. Wessman, M. Morin, K. Reijmar and K. Edwards, J. Colloid Interface Sci., 2010, 346, 127-135.

25 W. Jencks, Protein Sci., 1994, 3, 2459-2464.

26 A. C. Satterthwait and W. P. Jencks, J. Am. Chem. Soc., 1974, 96, 7018-7031.

27 M. Monette, M. R. Van Calsteren and M. Lafleur, Biochim. Biophys. Acta, Biomembr., 1993, 1149, 319-328.

28 T. Pott and E.-J. Dufourc, Biophys. J., 1995, 68, 965-977. 